\title{
CLOUD COMPUTING AND ACPI An approach to implement better energy management towards green IT
}

\author{
Y. Shabber Ali ${ }^{1}$, Ch. Pradeep Reddy ${ }^{2}$, Prof. M.Rajasekhara Babu ${ }^{3}$, \\ A.P.Sivakumar ${ }^{4}$ \\ ${ }^{I}$ BIT Institute of Technology, Hindupur, Andhra Pradesh, India \\ ${ }^{2}$ School of Information Technology Engineering, VIT University, Vellore, India \\ ${ }^{3}$ School of Computing Science and Engineering, VIT UNIVERSITY, Vellore, India \\ ${ }^{4}$ DEPARTMENT OF CSE, JNTU College of Engineering, Anantapur, A.P., India
}

\begin{abstract}
Cloud computing has proved to be a major breakthrough in terms of green computing. Cloud computing is one of the few technologies which offers the possibility of reducing energy consumption and carbon releases with almost negligible disturbance to business activities.The positives of this technology in terms of servicing and power consumption are apparent. In spite of all the positives, there are speculations about the efficiency of energy management in cloud computing. In this paper, we try to suggest a possible alternative to the various power saving techniques in cloud computing. The solution suggests implementation of ACPI (Advanced Configuration and Power Interface) in cloud servers. The various power states can be embedded in servers and switches to use optimal power when needed. Since switching is almost done all the time, this solution can help in reduction of significant power consumption during switching. The states may include sleep, idle and active. The proposed solution is supposed to reduce power consumption by activating the servers in the cloud which are in-demand. The servers not currently in use would be either in sleep or idle mode based on the probability at which requests are made.
\end{abstract}

Keywords: cloud; ACPI; power; servers; switching

\section{INTRODUCTION}

Due to the enormous scaling of the web in the past few years, several issues such as supporting innumerable online transactions or processing queries have arisen. To meet this ever increasing demand, companies like amazon, google and e-bay have responded by setting up huge data centers which encumber large number of servers and related infrastructure for storage, networking and cooling purposes [1].

Cloud computing represents a commercialization of these developments. Prior to the concept of cloud computing, the cost of acquiring resources was a costly task for organizations and individuals as well. But with the advent of cloud computing it has become a likely proposition [2]. Organizations and individuals can now acquire these resources by mere payment based on the services desired which also proves to be an easy source of income for the providers. It has therefore become easier to share and access data that was earlier beyond possible. Also, organizations can now concentrate on their core work system and competencies without bothering about the issues such as maintenance, administration and computing infrastructure [3].

Cloud computing can indeed save energy when it leads simply to the consolidation of servers. But looking at three different applications of cloud computing — storage, software and processing, it's clear that the energy efficiency savings are negated in a variety of scenarios. For example, one such instance is using cloud computing for storing data, and when the number of downloaded and accessed files becomes larger, those energy efficiency gains are erased.

\section{RECENT STUDIES}

Analysis sponsored by Microsoft reiterates that computing is most efficiently when concentrated in the cloud at giant data centers. Accenture and sustainability consulting company WSP Energy \& Environment conducted a study that revealed that the range of savings is between 30 percent and 90 percent from having hosted vs. on-premise IT infrastructure.

Its because of the nature cloud computing that there has been a reduction in the usage of energy which was very necessary because big corporations like Microsoft, Amazon and Google run enormous data centers 
delivering software over the internet. The credit goes to cloud computing because of its energy-saving technology that includes virtualization and dynamically provisioned software [3].

Furthermore, studies have been also done to compare products on premise and cloud based services. These studies suggest that a significant share of the company's operational expense is spent on IT by the cloud providers--much more than what a typical corporation spends on its own IT department. This condition hints an increase in the cost and efficiency improvement, which optimizes the data center and application performance to a level that many businesses cannot achieve on their own.

Naturally its very clear that a shared infrastructure for computing is going to be more efficient than corporate IT data centers that traditionally devote particular servers and storage to distinguish between applications in order to make them more reliable. The corporate data centers could be run very efficiently if there is a state of the art design for a data center and reduction of the consumption of energy is focused upon. In case of applications where there is a need to transport large amount of data, it is better to have on premise computing from an energy perspective [4].

In contrast, several studies have found that while cloud computing can deliver a more efficient use of computing power, in some cases, cloud computing can actually lead to more energy use than traditional inoffice computing [5].

Web companies have been embracing cloud computing in order to buy flexible, lower cost, on-demand computing power from companies like Amazon [4]. So cloud computing generally replaces the computing that would have been done by companies' own in-house computing resources. Simply put, data traveling to and from the cloud to the user's device (often times a computer) still requires energy. Most researchers overlook this fact in lieu of focusing solely on the energy consumed within the data center [6]. While the energy required to transport data from the cloud to each device is nominal on an individual level, across thousands and millions of devices it can add up; in certain cases, transporting the data actually overtakes any efficiencies gained by putting applications in the cloud [7].

Apparently, from the many studies conducted on cloud computing and power consumption, we can conclude that there is still a bit of speculation when it comes to power consumption in huge data centers and transportation of resources between client and the cloud. The topic will be continued in later sections where the proposed solution to further reduce power consumption by implementing the concept of ACPI which is widely used in PCs now a days.

\section{POWER CONSUMPTION IN CLOUD}

Table I. Summary of Cloud Services [8]

\begin{tabular}{|c|c|c|c|}
\hline & $\begin{array}{lll}\text { Software as a } \\
\text { Service }\end{array}$ & Storage as a Service & Processing as a Service \\
\hline Processing Location & Cloud & Client & $\begin{array}{l}\text { Short tasks at client, large } \\
\text { tasks in Cloud }\end{array}$ \\
\hline Storage Location & Cloud & Cloud & Client \\
\hline Transport Function & $\begin{array}{l}\text { Transmit } \\
\text { commands and } \\
\text { receive results }\end{array}$ & All files/documents & Files for large tasks \\
\hline
\end{tabular}

Table II. Hardware in Model of User Equipment

\begin{tabular}{|c|c|c|c|}
\hline Equipment & Parts & Power Consumption & \\
\hline & & Idle & Full Load \\
\hline $\begin{array}{l}\text { Modern mid-range } \\
\text { computer }\end{array}$ & $\begin{array}{l}\text { Intel E5200@2.5 GHz, } 2 \\
\text { GB RAM }\end{array}$ & $70 \mathrm{~W}$ & $110 \mathrm{~W}$ \\
\hline Old mid-range computer & $\begin{array}{l}\text { Intel Pentium } 4 @ 2.86 \\
\text { GHz, } 1 \text { GB RAM }\end{array}$ & $125 \mathrm{~W}$ & $210 \mathrm{~W}$ \\
\hline $\begin{array}{l}\text { Modern } \\
\text { computer }\end{array}$ & $\begin{array}{l}\text { Intel Q6600@2.4 GHz, } \\
\text { 4 GB RAM }\end{array}$ & $110 \mathrm{~W}$ & $175 \mathrm{~W}$ \\
\hline Low-end laptop & $\begin{array}{l}\text { Intel Atom @ } 1.6 \mathrm{GHz}, 2 \\
\text { GB RAM }\end{array}$ & $18 \mathrm{~W}$ & $18 \mathrm{~W}$ \\
\hline Terminal & ---- & $8 \mathrm{~W}$ & $8 \mathrm{~W}$ \\
\hline
\end{tabular}


Table II lists a range of commonly used classes of computers that users may use for personal computing and/or to access cloud computing services [8].

It has been claimed that transporting data between data centers and home computers can consume even larger amounts of energy than storing it [7]. Some papers which claim that energy consumption in cloud computing is less comparatively fail to state the fact that a significant amount of energy is wasted during transportation of information from a cloud to client. The data transport consumption is much larger in the public cloud processing services when compared to the private cloud services, especially when it comes to the high usage levels. This difference is mainly because of the fact that excessive number of router hops required on the public internet increases the consumption of energy in transport by a big margin while in the case of private cloud processing fewer routers are required [7]. This point hasn't been raised often as other positive features of cloud computing overshadow it. Another concern is particularly when the user device is mobile where battery life is an important. Also, as the scope of cloud services grows it will result in the increased consumption of energy. It has been found that power consumption by clouds contribute to the production of Green house gases which is likely to increase every year. It is therefore important to take measures to reduce this contribution to increase of green house gases and promote greener IT. One such strategy which we suggest in this paper is to embed features of ACPI in clouds. As said above, this might help in further reduction of power consumption by clouds. Transportation cost can be also reduced by enabling desired servers to communicate and disabling the rest which are not needed at a specific time. This supposition can be strengthened by the fact that whenever the usage of cloud service is infrequent and at low intensity, less power is consumed in cloud computing when compared to the conventional computing techniques [7]. The reason is that at low usage levels, power consumption for storage dictates the total power consumption, and there is minimal power consumption for transport. When it comes to the medium and high level usage levels, the energy required to transport data is more so that the total power consumption is dominated by the transport and the overall energy consumption is increased. Power for transport can be as low as $10 \%$ and $25 \%$ at low usage levels for private and public storage services, respectively, and nearly $60 \%$ and $90 \%$, respectively, at high usage levels [7]. An important factor which determines rate of energy consumption is the number of users per server. Therefore, the server with maximum users needs to be kept in Active state often whereas other servers can be put to idle state to minimize consumption of power. These states correspond to states of ACPI [9]. This is discussed in sections ahead.

\section{ACPI: A SOLUTION}

To understand the significance of the solution it is important to know what ACPI is and how can it help in reduction of power consumption in clouds. ACPI, which stands for Advanced Configuration and Power Interface is an open industry standard which has a key feature of power management.

This type of power management allows the operating system running on a computer to control the power. Prior to this standard, the power management was put into the hands of the computer's BIOS, which meant that the operating system was unable to perform tasks such as physically turning off your computer once you have performed a shutdown. ACPI allows the operating system to do this as well as many other aspects of the power savings such as turning off the monitor after a certain amount of time and turning off the hard disks when they have been inactive for a predetermined number of minutes. The solution we suggest is to use this feature of ACPI to control power usage in cloud servers where servers not in demand can be temporarily turned off to reduce the energy which otherwise would have been uselessly wasted had they been turned on.

Since the advent of ACPI, power saving features that were once only found in portable computers, have become commonplace on desktop computers and servers as well. Using these features, a cloud may be put into a state in which very few servers are powered on, but can be quickly awaken in the case of a request/demand such as request of a resource which resides on that server by a client. Compatibility is a key issue when it comes to ACPI. There are many different states used to define different situations in ACPI. There are global states that cover the different power states of the system as a whole. What we suggest here in this paper is to implement states like Active, idle and sleep which are analogical to states in any normal PC. The first step can be judging the amount of requests each server in the cloud receives. Based on this information, each server can be made to go into Active, idle or sleep state as and when required. When a server is needed it would stay active. As soon as the request is completed the server shall go back to idle state if the probability of receiving another request shortly is high. If not, then the server can temporarily go into sleep state and be able to quickly awake as soon as any request is received. In this way, each server would consume energy only when it is processing requests. This will save the energy which is inappropriately used when servers stay active all the time anticipating requests to occur. Thinking about all the positives, it is quite apparent that the suggested solution would definitely help reduce energy consumption in clouds and can guarantee greener IT without disrupting the services. All in all, ACPI can prove to be a breakthrough solution in clouds.

\section{REFERENCES}

[1]. Jack Dennis (MIT), Guang R. Gao, and Xiaoxuan Meng (Univ. of Delaware), Experiments with the Fresh Breeze Tree-Based Memory Model, ISC 2011 
[2]. http://www.uic.edu

[3]. http://www.umiacs.umd.edu

[4]. Robert W. A. Ayre , Jayant Baliga, , Kerry Hinton, Rodney S. Tucker Green Cloud Computing: Balancing Energy in Processing, Storage and Transport Proceedings of the IEEE, Vol. 99, No. 1. (January 2011), pp. 149167.doi:10.1109/JPROC.2010.2060451 Key: citeulike:8015489

[5]. http://www.launchpad.iglou.com

[6]. http://www.www.topix.net

[7]. http://www.www.mrrotten.com

[8]. http://www.www.physorg.com

[9]. Jayant Baliga, Robert W. A. Ayre, Kerry Hinton, Rodney S. TuckerGreen Cloud Computing: Balancing Energy in Processing, Storage and Transport Proceedings of the IEEE, Vol. 99, No. 1. (January 2011), pp. 149167.doi:10.1109/JPROC.2010.2060451 Key: citeulike:8015489

[10]. http://www.cs.uccs.edu 